Herzschr Elektrophys $2021 \cdot 32: 293-294$ https://doi.org/10.1007/s00399-021-00798-9 Angenommen: 5. Juli 2021

(c) Springer Medizin Verlag $\mathrm{GmbH}$, ein Teil von Springer Nature 2021

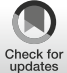

\section{Autonome Innervation des Herzens}

\author{
Rainer Schimpf' · Thomas Klingenheben ${ }^{2}$ \\ 'Kardiologische Praxisklinik Ludwigshafen, Neustadt a.d. Weinstraße, Deutschland \\ ${ }^{2}$ Praxis für Kardiologie Bonn u. Ambulante Herzkatheter-Kooperation Bonn, Bonn, Deutschland
}
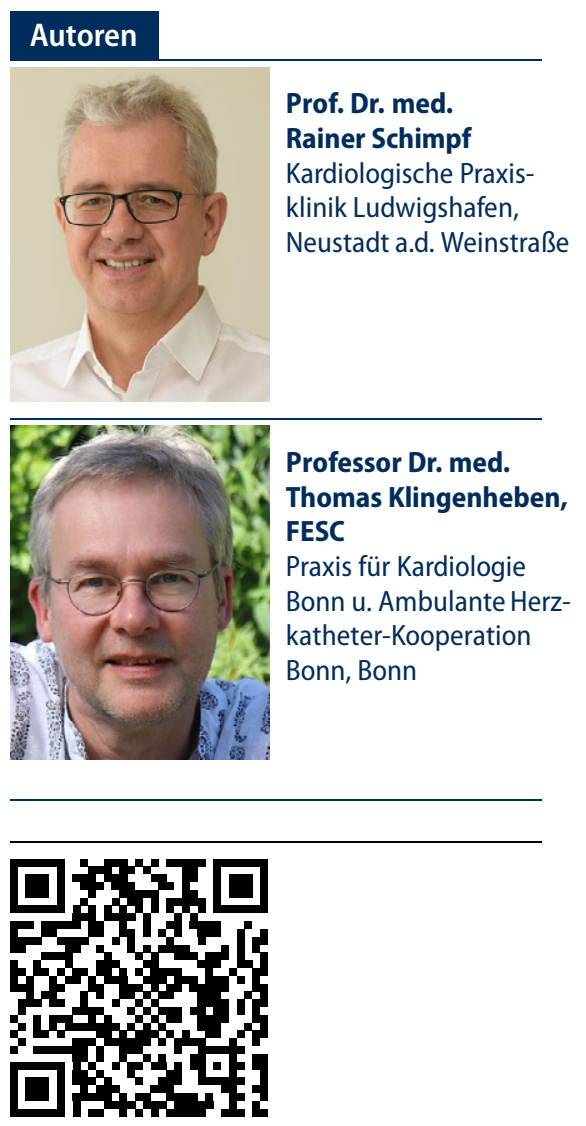

QR-Code scannen \& Beitrag online lesen
Liebe Leserin, lieber Leser,

täglich vollzieht sich ca. 100.000 Mal der geregelte Ablauf der elektromechanischen Aktivierung der kardialen Kontraktion. Initiiert von rhythmischen spontanen Depolarisationen der Sinusknotenzellen mit Weiterleitung über das spezifische Reizleitungssystem erfolgt schließlich der AVsequenzielle Auswurf in den kleinen und großen Blutkreislauf. Mit einer enormen Präzision und Stabilität arbeitet dieses intrakardiale Aktivierungssystem. Der mittlerweile erhebliche Erkenntniszuwachs der letzten Jahre führt uns vor Augen, dass dieser Ablauf weitaus komplexer ist, als man zu Beginn der Erforschung des Herzrhythmus vermutete. So wird die beschriebene Aktivierung des Herzschlags durch Einflüsse des intrinsischen kardialen autonomen Nervensystems (ANS), bestehend aus epikardialen Ganglien, („epicardial ganglionated plexus" und "fat pads"), die untereinander mit interagierenden neuronalen Netzwerken in Verbindung stehen, erweitert. Hinzu kommt das extrinsische ANS, welches mit sympathischen Nervenfasern und dem N. vagus, deren Interaktion mit dem Hirnstamm sowie kardialen präganglionären Fasern regulatorisch wirkt.

Störungen dieses komplexen Systems der elektromechanischen Aktivierung können sowohl durch intrakardiale strukturelle Veränderungen und Erkrankungen (Myokardinfarkt, Narbe, Dilatation, Inflammation), elektrische Erkrankungen (z.B. primär elektrische Erkrankungen des Herzens), aber auch infolge primär extrakardialer Ursachen (z. B. autonome Erkrankungen als Folge eines Diabetes mellitus, neurologische Erkrankungen, posturales Tachykardiesyndrom etc.) auftreten.
Wir freuen uns sehr, dass wir für das Themenheft zur autonomen Innervation des Herzens zahlreiche international renommierte Autoren gewinnen konnten.

Mark Shen (Chicago/USA) gibt eine exzellente Einführung und einen Überblick über Anatomie und Funktion der extrinsischen und intrinsischen Komponenten der kardialen autonomen Innervation.

Dass einzelne Komponenten der autonomen kardialen Innervation auch Therapieziel im Rahmen interventioneller Behandlungsstrategien darstellen können, wird in der Übersicht von Stefan Ailoaei aus der Arbeitsgruppe von Sabine Ernst (London/England) eindrucksvoll dargestellt. Am Beispiel der klinisch häufigsten Rhythmusstörung Vorhofflimmern werden die Ansätze zur Lokalisation und Modulation des ANS erläutert.

Die Bedeutung der Aktivität des ANS in der Genese des arrhythmogenen plötzlichen Herztods ist in den letzten 50 Jahren im Kontext von sowohl fortgeschrittenen strukturellen Herzerkrankungen als auch primär elektrischen Erkrankungen vielfach untersucht worden. Philippe Maury (Toulouse/Frankreich) hat den wissenschaftlichen Kenntnisstand zur sympathischen und parasympathischen Beeinflussung von primären Arrhythmopathien/ lonenkanalerkrankungen systematisch aufbereitet.

Die Nutzbarmachung von EKG-basierten Markern der kardialen ANS-Aktivität zur Arrhythmie-Risikostratifikation ist bei strukturellen Herzerkrankungen am besten untersucht. Beatrice de Maria aus der Arbeitsgruppe von Maria Teresa La Rovere (Mailand u. Pavia/Italien) fasst den diesbezüglichen Kenntnisstand zur Wertigkeit der Herzfrequenzvariabilität aus 24-h-Langzeit-EKG-Registrierungen zu- 
sammen. Heikki Huikuri (Oulu/Finnland) weitet den Blick der ANS-basierten Risikostratifikation des plötzlichen Herztods und stellt neuere EKG-basierte Parameter vor. Zudem diskutiert er kritisch die Gründe, weshalb bislang keiner dieser Marker in der klinisch-kardiologischen Routine etabliert werden konnte.

Ein Beispiel für die Komplexität mutmaßlich primär extrakardialer Effekte auf eine veränderte Herzschlagfolge und Störung der zerebralen Autoregulation stellt das posturale orthostatische Tachykardiesyndrom (POTS) dar. Der Leidensdruck dieser häufig junge Frauen betreffenden Erkrankung ist teilweise erheblich, und die therapeutischen Optionen dieses Krankheitsbilds sind limitiert. Eine invasive elektrophysiologische Behandlungsmöglichkeit klinisch schwerer therapierefraktärer Fälle stellt Cinzia Monaco aus der Arbeitsgruppe von Carlo de Asmundis (Brüssel/Belgien) im vorliegenden Schwerpunkt vor. Zur Vertiefung der Grundlagen des POTS sei der interessierte Leser auf den Artikel von Mohammed Ruzieh und Blair P. Grubb in Herzschr Elektrophys 2018;29:183-18 verwiesen.

Ralph Bosch (Ludwigsburg) ist Koautor des DGK-Kommentars zu den ESC Synkopen-Leitlinien und diskutiert die wichtigsten pathophysiologischen, diagnostischen und therapeutischen Aspekte der durch das ANS vermittelten Reflexsynkopen.

Dass auch primär neurogene autonome Erkrankungen eine symptomatische kardiovaskuläre Beteiligung aufweisen, wird von Christina Haubrich (Düsseldorf) und Thomas Klingenheben (Bonn) exemplarisch anhand der primären autonomen Dysfunktion („pure autonomic failure ${ }^{\prime \prime}$ ) dargestellt.

Die Heftherausgeber hoffen, mit der Auswahl der Themen Ihr Interesse geweckt zu haben, und wünschen eine anregende Lektüre.

Ihre

Rainer Schimpf und Thomas Klingenheben

\section{Korrespondenzadresse}

Prof. Dr. med. Rainer Schimpf

Kardiologische Praxisklinik Ludwigshafen Schütt 2, 67433 Neustadt a.d. Weinstraße, Deutschland

rainer.schimpf@kardiologie-ludwigshafen.de

Professor Dr. med. Thomas Klingenheben, FESC

Praxis für Kardiologie Bonn u. Ambulante Herzkatheter-Kooperation Bonn Im Mühlenbach 2 B, 53127 Bonn, Deutschland Klingenheben@aol.com

Interessenkonflikt. R. Schimpf und T. Klingenheben geben an, dass kein Interessenkonflikt besteht. 